\title{
Niveles de ansiedad y su relación con los trastornos temporomandibulares en individuos jóvenes estudiantes de estomatología de la Universidad Alas Peruanas
}

\section{Levels of anxiety and their relationship with the dysfunctions temporomandibulares in young studying individuals of stomatology of the University Alas Peruanas}

\author{
Melvin Cisneros del Águila*
}

http://dx.doi.org/10.21503/CienciayDesarrollo.2010.v12.03

\section{RESUMEN}

El estudio tiene por objetivo determinar la relación entre la ansiedad y los trastornos temporomandibulares en individuos jóvenes estudiantes de estomatología. Se examinaron 80 estudiantes entre 18 y 30 años. Se les aplicó el test de IDARE y el índice de Helkimo, para medir los niveles de ansiedad y su asociación con los TTM, teniendo en consideración el género y la edad. La asociación entre la ansiedad, el género y la edad no fue significativa. La prevalencia de los TTM fue de $95 \%$; la severidad, de grado leve $(58,8 \%)$, moderado $(31,3 \%)$, severo (5\%). Los niveles de ansiedad en relación a los TTM fueron significativos: ansiedad-estado, ansiedad-rasgo y ansiedad rasgo-estado, con los grados de TTM leve, moderado y severo, en los rangos de $\mathrm{p}=0,031, \mathrm{p}=0,20, \mathrm{p}=0,020(\mathrm{p}<0,05)$. Además, individuos con niveles severos de ansiedad evidenciaron $100 \%$ de TTM; $95 \%$ de sujetos con TTM presentaron ansiedad rasgo y ansiedad rasgo-estado moderado.

Palabras clave: desórdenes temporomandibulares, oclusión, dolor orofacial.

\section{ABSTRACT}

The objective of the study is to determine the relation between the anxiety and the Temporomandibular disorder in young individual stomatology students. 80 students aged between 18 and 30 were examined, applied to the test of IDARE, and the index of Helkimo to measure the levels of anxiety and their association with TMD taking into consideration the gender and age. The association between the anxiety, the gender, and age was not significant. The prevalence of TMD was of $95 \%$; severity, slight degree $(58,8 \%)$, moderate $(31,3 \%)$, severe $(5 \%)$. The levels of anxiety in relation to the TDM were significant: Anxiety- been, anxiety-characteristic and anxiety characteristic-been, with the TDM degrees light, moderate and severe, in the ranks of $\mathrm{p}=0,031, \mathrm{p}=0,20, \mathrm{p}=0,020(\mathrm{p}<0,05)$. In addition, individuals with severe levels of anxiety, demonstrated $100 \%$ of TDM; $95 \%$ of subjects with TDM displayed anxiety characteristic and anxiety characteristic-been moderate.

Key words: temporomandibular disorders, occlusion, orofacial pain.

\footnotetext{
* Profesor de prostodoncia parcial removible y clínica estomatológica. Universidad Alas Peruanas. Maestro en investigación y docencia universitaria, doctor en odontología, especialista en rehabilitación oral.
} 


\section{INTRODUCCIÓN}

La considerable agitación del ritmo de vida actual hace que gran parte de la población se encuentre sometida constantemente a la acción de estresores, encontrándose manifestaciones de ello en diferentes órganos de la economía humana. Una de estas manifestaciones, como respuesta del organismo a esta injuria, se refleja en el sistema estomatognático, conociéndose esta serie de signos y síntomas como desórdenes o trastornos temporomandibulares (TTM). Los síntomas y signos asociados a los TTM tienen una incidencia y una prevalencia muy elevada en la población. Entre un 15 y $74 \%$ presentan al menos un síntoma, y de un 33 a $86 \%$ presentan un signo clínico, resultando un promedio de $45 \%$ de sujetos que presentan un síntoma y un $58 \%$ que presenta un signo clínico. En la actualidad, existe consenso respecto de que los trastornos temporomandibulares tienen un origen multifactorial. Se han identificado como factores de riesgo las condiciones oclusales y las alteraciones psicológicas, ubicándose dentro de este último rubro aspectos fisiopatológicos relacionados con la ansiedad.

Castillo, R. et al., en un trabajo realizado en La Habana, en una muestra de 100 individuos, estudiaron los niveles de ansiedadrasgo y ansiedad-estado en relación con los hábitos parafuncionales y la disfunción temporomandibular. Encontraron que la ansiedad-estado presentó una asociación altamente significativa con los TTM: $52 \%$ en el grupo experimental y $70 \%$ en el grupo control. Además, concluyen que los individuos con TTM tienen niveles más altos de ansiedad-rasgo que los sanos. Bonjardim et al., en un estudio cuya meta era verificar el predominio de la ansiedad y depresión en adolescentes y su relación con los signos y síntomas de los TTM, encontraron, en una muestra de 217 adolescentes entre 12 y 18 años, que la ansiedad y la depresión estuvieron presentes en el $16,58 \%$ y $26,71 \%$ de los casos respectivamente. Concluyen que la ansiedad y la depresión, aunque de intensidad suave, son comunes en adolescentes. Ambos procesos fueron asociados a un aumento del número de síntomas de TTM.John, Mike T. et al. estudiaron el nivel de debilitamiento de la calidad de vida en pacientes con TTM; la muestra estuvo conformada por 416 individuos. El estudio concluye que el nivel de debilitamiento de la calidad de vida relativa a la salud oral se halla marcadamente deteriorada en pacientes con TTM. Ortiz, F., en un trabajo de investigación realizado en la Facultad de Odontología de la Universidad de San Martín de Porres, sobre una muestra de 94 alumnos entre 18 y 25 años, aplicando el test de IDARE y el índice de Helkimo, encontró que la prevalencia de ansiedad se concentró en el nivel leve, tanto para la ansiedad-estado como para la ansiedadrasgo. En relación con el género, en el femenino encontró un nivel severo de ansiedad (36,8 \%), en comparación con el masculino, que evidenció $7,7 \%$ de ansiedad severa. La prevalencia de los trastornos temporomandibulares fue de $87,2 \%$ de la muestra; el grado de severidad fue la disfunción leve $(73,4 \%)$; los signos más prevalentes fueron los ruidos articulares (59,6\%), limitación en el movimiento $(39,4 \%)$ y desviación mandibular $(38,3 \%)$. El síntoma más prevalente fue el dolor a la palpación muscular (43,6 \%). No encontró diferencias significativas en la presencia de TTM según el género. Tampoco encontró diferencias estadísticamente significativas para la presencia de TTM, según los niveles de ansiedad, ansiedadestado, ansiedad-rasgo. 1,2, 3,4

Las situaciones estresantes pueden desencadenar TTM asociados a factores predisponentes como la desarmonía, que puede existir en la oclusión del individuo. En relación con este aspecto, Bell, W., citado por Molina, manifiesta que el papel de la tensión es de gran importancia, considerando como el principal activador de la desarmonía oclusal a la tensión 
emocional, que actúa de diversas formas, entre las cuales está acrecentar el bruxismo y otros hábitos parafuncionales, aumentando la actividad muscular general que conduce a la aparición de los primeros signos y síntomas de los TTM. 5, 6

Los TTM constituyen una serie de desarmonías morfofuncionales de los componentes del aparato masticatorio, cuyas características engloban la presencia de ciertos signos y síntomas relacionados con el dolor y problemas en la ATM. Schulte, González y Greene adicionan a estos signos y síntomas la presencia de hábitos parafuncionales como el bruxismo ${ }^{7,8,9}$. En la actualidad, se puede afirmar en forma categórica que la etiología de los TTM responde a un origen multifactorial. En este contexto, McNeill, Pullinger y Seligman en el 2000 realizaron estudios prometedores tomando en consideración las relaciones dinámicas oclusales, y con lo estudiado por Laskin y Moulin comienzan a defender la naturaleza factorial de los TTM. ${ }^{10,11,12,13,14}$ De acuerdo con Irving, J., aproximadamente el $80 \%$ de la población general tiene por lo menos un signo clínico de disfunción articular, y alrededor del $33 \%$ tiene un síntoma. Se conoce que los TTM son la causa más común de dolor facial después del dolor dental, y que puede afectar hasta el $15 \%$ de la población general. ${ }^{15}$ Estudios epidemiológicos y clínicos realizados por Agerber y Carlsson, Agerber y Osterberg, demostraron que más del $50 \%$ de la población adulta padecía el mismo signo de disfunción de ATM. Del mismo modo, se conoce que los TTM afectan con mayor frecuencia al sexo femenino en una relación de 4 a 1 y de 2 a 1 en edades de 25 a 35 años, siendo la franja etárea predominante en ambos sexos de 20 a 40 años. Cabe mencionar que en todos los casos no es necesario realizar un tratamiento, pues solo del 5 al $6 \%$ de los afectados lo necesitan, siendo la disminución de la apertura mandibular la más reportada. $16,17,18,19,20$
El objetivo general se centró en determinar la relación que existe entre los niveles de ansiedad y los diferentes grados de trastornos temporomandibulares en individuos jóvenes estudiantes de estomatología.

En el caso de los objetivos específicos, se tomaron en consideración los siguientes:

a) Identificar los niveles de ansiedad de acuerdo a la edad y el género.

b) Determinar la prevalencia y severidad de los trastornos temporomandibulares.

c) Identificar las variaciones en cuanto al grado y distribución de los trastornos temporomandibulares de acuerdo a la edad y el género.

d) Identificar los signos y síntomas asociados a los trastornos temporomandibulares en función del género.

e) Determinar la presencia de ansiedad en relación con los signos y síntomas más prevalentes de los trastornos temporomandibulares.

Hipótesis general. Se planteó que sí existe relación significativa entre los niveles de ansiedad y los diferentes grados de trastornos temporomandibulares en individuos jóvenes estudiantes de estomatología

Hipótesis Secundarias. Se plantearon las siguientes:

a) Los niveles de ansiedad de acuerdo a la edad y el género son de una relativa significancia.

b) Sí existen variaciones en relación con la prevalencia y severidad de los trastornos temporomandibulares.

c) Sí existen variaciones en cuanto al grado y distribución de los trastornos temporomandibulares de acuerdo a la edad y el género.

d) Sí existen variaciones en relación con la presencia de signos y síntomas de los trastornos temporomandibulares en función del género. 
Material y método. Corresponde a un estudio clínico, prospectivo, transversal y analítico.

Población. Estuvo conformada por 400 alumnos del V al IX ciclo de la Escuela AcadémicoProfesional de Estomatología de la Facultad de Ciencias de la salud de la Universidad Alas Peruanas

Muestra. Se utilizó un muestreo probabilístico no intencional, seleccionando 80 estudiantes que cumplieron con los criterios de inclusión y exclusión.

Técnicas e instrumentos. Para lleva a efecto la investigación se utilizó la observación clínica estomatológica, odontograma, Índice de Helkimo y el Test de IDARE.

\section{RESULTADOS}

Se estudiaron los niveles de ansiedad rasgoestado según el género y grupo etáreo, además de la prevalencia, severidad, grado, signos y síntomas asociados a los TTM según género y grupo etáreo; finalmente, se consideró la relación entre los niveles de ansiedad y los TTM.

Los niveles de ansiedad en la muestra estudiada nos indican que los niveles leve y severo de ansiedad son los más predominantes en la muestra, para los valores de ansiedad-estado, ansiedad-rasgo y ansiedad rasgo-estado; ansiedad estado leve: 37,5 $\%$, severo: $36,25 \%$; ansiedad-rasgo leve: $43,75 \%$, severo: $31,25 \%$; ansiedad rasgo-estado leve: $45 \%$, severo: $30 \%$. (Ver Tabla No 1 )

En la ansiedad rasgo-estado según el género, el sexo femenino (17 individuos: 35,4\%) presenta una tendencia mayor que el masculino (7 individuos: $21,9 \%$ ) a desarrollar un nivel severo de ansiedad. (Ver Tabla No 2)

En la ansiedad rasgo-estado según el grupo etáreo, se encuentra una pequeña tendencia hacia una ansiedad rasgo-estado severa en los

Tabla 1. Niveles de ansiedad en la muestra estudiada

\begin{tabular}{|c|c|c|c|c|c|c|c|c|c|c|}
\hline & \multicolumn{2}{|c|}{$\begin{array}{c}\text { Ansiedad } \\
\text { estado }\end{array}$} & \multicolumn{2}{|c|}{$\begin{array}{c}\text { Ansiedad } \\
\text { rasgo }\end{array}$} & \multicolumn{2}{|c|}{$\begin{array}{c}\text { Ansiedad } \\
\text { rasgo-estado }\end{array}$} & \multicolumn{2}{|c|}{$\begin{array}{c}\text { Estado } \\
\text { preocup. }\end{array}$} & \multicolumn{2}{|c|}{$\begin{array}{r}\text { Estado } \\
\text { satisfac. }\end{array}$} \\
\hline & No. & $\%$ & $\mathrm{~N}^{\circ}$. & $\%$ & $\mathrm{~N}^{\circ}$. & $\%$ & $\mathrm{~N}^{\circ}$. & $\%$ & $\mathrm{~N}^{\circ}$. & $\%$ \\
\hline Muy leve & 0 & 0 & 0 & 0 & 0 & 0 & 14 & 17,5 & 20 & 25 \\
\hline Leve & 30 & 37,5 & 35 & 43,75 & 36 & 45 & 42 & 52,5 & 32 & 40 \\
\hline Moderado & 21 & 26,25 & 20 & 25 & 20 & 25 & 19 & 23,75 & 22 & 27,5 \\
\hline Severo & 29 & 36,25 & 25 & 31,25 & 24 & 30 & 5 & 6,25 & 6 & 7,5 \\
\hline Total & 80 & 100 & 80 & 100 & 80 & 100 & 80 & 100 & 80 & 100 \\
\hline
\end{tabular}

Tabla 2. Niveles de ansiedad rasgo-estado según género

\begin{tabular}{llc|c|c|c} 
& & \multicolumn{4}{c}{ Sexo } \\
\cline { 3 - 6 } & & \multicolumn{2}{c}{ Masculino } & \multicolumn{2}{c}{ Femenino } \\
\cline { 3 - 7 } & & Recuento & $\begin{array}{c}\text { \% del N de la } \\
\text { columna }\end{array}$ & Recuento & $\begin{array}{c}\text { \% del N de la } \\
\text { columna }\end{array}$ \\
\hline $\begin{array}{l}\text { Ansiedad } \\
\text { rasgo- }\end{array}$ & Leve & 17 & $53,1 \%$ & 19 & $39,6 \%$ \\
estado & Moderado & 8 & $25,0 \%$ & 12 & $25,0 \%$ \\
& Severo & 7 & $21,9 \%$ & 17 & $35,4 \%$ \\
\hline & Total & 32 & $100,0 \%$ & 48 & $100,0 \%$ \\
\hline
\end{tabular}


Tabla 3. Niveles de ansiedad rasgo - estado según grupo etáreo

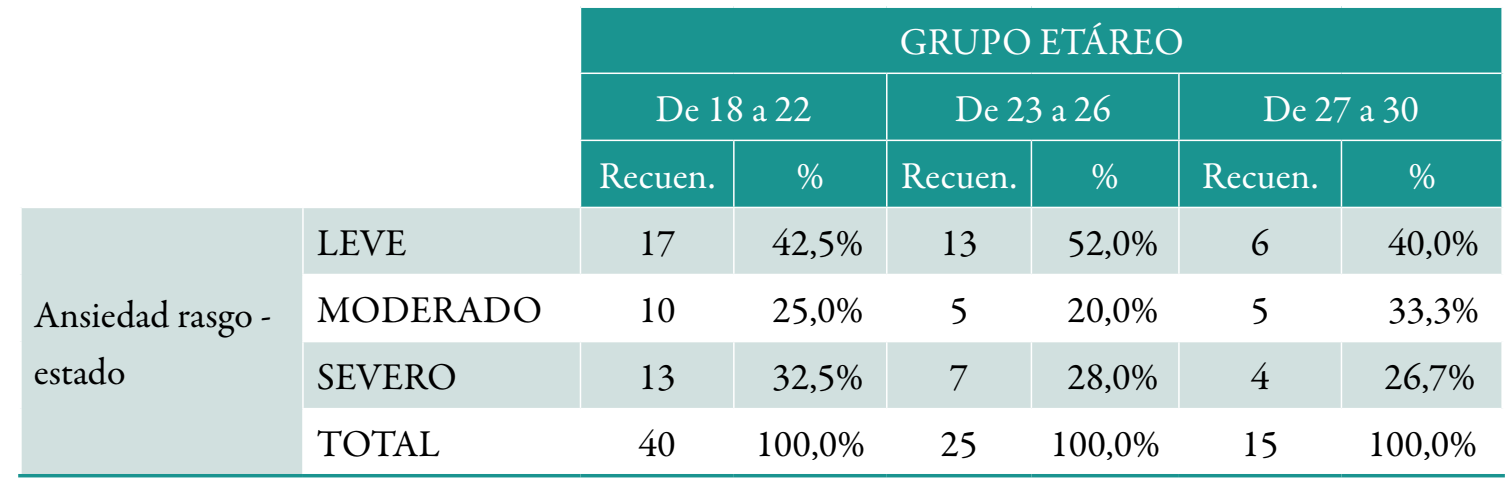

Tabla 4. Prevalencia de los TTM

\begin{tabular}{|l|c|c|}
\hline & $N^{\circ}$. & $\%$ \\
\hline Sanos & 4 & 5 \\
\hline Con TTM & 76 & 95 \\
\hline TOTAL & 80 & 100 \\
\hline
\end{tabular}

grupos etáreos de 18 a 22 años (13 individuos: $32,5 \%)$ y de 23 a 26 años (7 individuos: 28\%). (Ver Tabla No 3 )

En referencia a la prevalencia de los TTM, se encontraron 76 individuos afectados (95\%) y 4 $\operatorname{sanos}(5 \%)$. (Ver Tabla No 4 )

En relación con la severidad de los TTM, se encontraron 4 individuos $(5 \%)$ con función normal, 47 (58\%) con disfunción leve, 25 (31,3 \%) con disfunción moderada y 4 (5\%) con disfunción severa. (Ver Tabla No 5)

Respecto del grado de TTM según el género, la función normal predominó en el masculino (3 individuos: 9,4\%), la disfunción leve en el sexo femenino (30 individuos: 62,5\%), la disfunción moderada en el sexo masculino (11 individuos: $34,4 \%)$, y la disfunción severa en el sexo femenino (3 individuos: 6,3\%). (Ver Tabla № 6)

En relación con el grado de TTM según el grupo etáreo, los tres grupos muestran una
Tabla 5. Severidad de los TTM

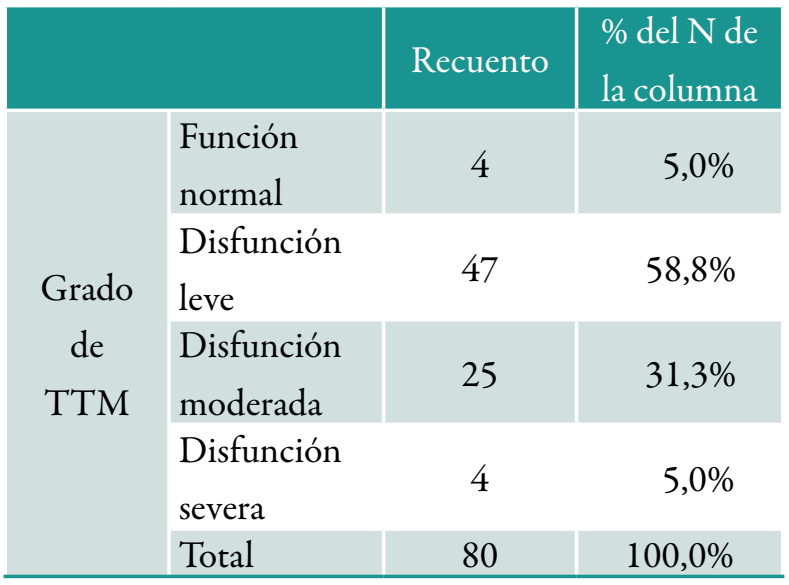

tendencia similar a disfunción leve y moderada; la disfunción severa se reparte en los tres grupos, con 2 (5\%), 1 (4\%) y 1 (6,7\%) afectados respectivamente. (Ver Tabla No 7 )

En cuanto a los signos y síntomas asociados a los TTM, los que más presentaron fueron: movilidad mandibular (75 individuos: 93,75\%), alteraciones de la función de la ATM (51 individuos: 63,75\%), dolor en los músculos masticatorios (26 individuos: 32,5\%). El dolor en el movimiento mandibular y dolor a la ATM fueron de menor cuantía. (Ver Tabla No 8)

En relación con los niveles de ansiedad-estado y los grados de TTM, las diferencias encontradas fueron estadísticamente significativas a la prueba de chi cuadrado $(p=0,031)(p<0,05)$, 
Tabla 6. Grado de TTM según el género

\begin{tabular}{|c|c|c|c|c|c|}
\hline & & \multicolumn{4}{|c|}{ SEXO } \\
\hline & & \multicolumn{2}{|c|}{ MASCULINO } & \multicolumn{2}{|c|}{ FEMENINO } \\
\hline & & Recuento & $\begin{array}{c}\% \text { del } \mathrm{N} \text { de la } \\
\text { columna }\end{array}$ & Recuento & $\begin{array}{c}\% \text { del } \mathrm{N} \text { de la } \\
\text { columna }\end{array}$ \\
\hline \multirow{5}{*}{ Grado de TTM } & Función normal & 3 & $9,4 \%$ & 1 & $2,1 \%$ \\
\hline & Disfunción leve & 17 & $53,1 \%$ & 30 & $62,5 \%$ \\
\hline & $\begin{array}{l}\text { Disfunción } \\
\text { moderada }\end{array}$ & 11 & $34,4 \%$ & 14 & $29,2 \%$ \\
\hline & Disfunción severa & 1 & $3,1 \%$ & 3 & $6,3 \%$ \\
\hline & Total & 32 & $100,0 \%$ & 48 & $100,0 \%$ \\
\hline
\end{tabular}

\section{Tabla 7. Grado de TTM según grupo etáreo}

\begin{tabular}{|c|c|c|c|c|c|c|c|}
\hline & & \multicolumn{6}{|c|}{ GRUPO ETÁREO } \\
\hline & & \multicolumn{2}{|c|}{ De 18 a 22} & \multicolumn{2}{|c|}{ De 23 a 26} & \multicolumn{2}{|c|}{ De 27 a 30} \\
\hline & & Recuen. & $\%$ & Recuen. & $\%$ & Recuen. & $\%$ \\
\hline \multirow{5}{*}{$\begin{array}{c}\text { GRADO } \\
\text { DE } \\
\text { TTM }\end{array}$} & Función normal & 1 & $2,5 \%$ & 2 & $8,0 \%$ & 1 & $6,7 \%$ \\
\hline & Disfunción leve & 24 & $60,0 \%$ & 14 & $56,0 \%$ & 9 & $60,0 \%$ \\
\hline & $\begin{array}{l}\text { Disfunción } \\
\text { moderada }\end{array}$ & 13 & $32,5 \%$ & 8 & $32,0 \%$ & 4 & $26,7 \%$ \\
\hline & $\begin{array}{l}\text { Disfunción } \\
\text { severa }\end{array}$ & 2 & $5,0 \%$ & 1 & $4,0 \%$ & 1 & $6,7 \%$ \\
\hline & Total & 40 & $100,0 \%$ & 25 & $100,0 \%$ & 15 & $100,0 \%$ \\
\hline
\end{tabular}

Tabla 8. Signos y síntomas asociados a los TTM

\begin{tabular}{|l|c|c|c|c|c|c|c|c|c|c|}
\hline & \multicolumn{2}{|c|}{ MOV. MAND } & \multicolumn{2}{|c|}{ FUNC. ATM } & \multicolumn{2}{c|}{ DOL. MUSC. } & \multicolumn{2}{c|}{ DOL. ATM. } & \multicolumn{2}{c|}{ DOLOR. MOV. } \\
\hline & $N^{\circ}$ & $\%$ & $N^{\circ}$ & $\%$ & $N^{\circ}$ & $\%$ & $N^{\circ}$ & $\%$ & $N^{\circ}$ & $\%$ \\
\hline NO & 5 & 6,25 & 29 & 36,25 & 54 & 67,5 & 69 & 86,25 & 58 & 72,5 \\
\hline SÍ & 75 & 93,75 & 51 & 63,75 & 26 & 32,5 & 11 & 13,75 & 22 & 27,5 \\
\hline TOTAL & 80 & 100 & 80 & 100 & 80 & 100 & 80 & 100 & 80 & 100 \\
\hline
\end{tabular}

encontrándose los tres niveles de ansiedadestado asociados a los diversos grados de TTM (leve, moderado y severo). De 50 individuos con niveles de ansiedad-estado altos (moderado y severo), 47 presentaron un grado de TTM. (Ver Tabla No 9) 


\section{Tabla 9. Niveles de ansiedad-estado según el grado de TTM}

\begin{tabular}{|c|c|c|c|c|c|c|c|}
\hline & \multicolumn{6}{|c|}{ ANSIEDAD-ESTADO } \\
\hline & & \multicolumn{2}{|c|}{ LEVE } & \multicolumn{2}{|c|}{ MODERADO } & \multicolumn{2}{|c|}{ SEVERO } \\
\hline & & Recuen. & $\%$ & Recuen. & $\%$ & Recuen. & $\%$ \\
\hline \multirow{5}{*}{ Grado de TTM } & Función normal & 1 & $3,3 \%$ & 3 & $14,3 \%$ & 0 & $0 \%$ \\
\hline & Disfunción leve & 13 & $43,3 \%$ & 12 & $57,1 \%$ & 22 & $75,9 \%$ \\
\hline & $\begin{array}{l}\text { Disfunción } \\
\text { moderada }\end{array}$ & 15 & $50,0 \%$ & 5 & $23,8 \%$ & 5 & $17,2 \%$ \\
\hline & Disfunción severa & 1 & $3,3 \%$ & 1 & $4,8 \%$ & 2 & $6,9 \%$ \\
\hline & Total & 30 & $100,0 \%$ & 21 & $100,0 \%$ & 29 & $100,0 \%$ \\
\hline
\end{tabular}

Pruebas de chi-cuadrado de Pearson 0,031 p<0,05 (SIGNIFICATIVO)

La relación entre los niveles de ansiedadrasgo y los grados de TTM, de acuerdo a la prueba de chi cuadrado, resultó estadísticamente significativa $(p=0,020) \quad(p<0,05)$. Se puede distinguir con claridad la asociación entre los tres niveles de ansiedad-rasgo y los diversos grados de TTM (leve, moderado y severo). De 45 individuos con niveles de ansiedad-rasgo altos (moderado y severo), 44 presentaron un grado de TTM. (Ver Tabla No 10)
La relación existente entre los niveles de ansiedad rasgo-estado y los grados de TTM, de acuerdo a la prueba de chi cuadrado, resultó estadísticamente significativa $(p=0,020)(p<0,05)$. Estos hallazgos nos llevan a percibir con certeza la asociación importante entre los tres niveles de ansiedad rasgo-estado y los diversos grados de TTM (leve, moderado y severo). De 44 individuos con niveles de ansiedad rasgo-estado altos

\section{Tabla 10. Niveles de ansiedad-rasgo según el grado de TTM}

\begin{tabular}{|c|c|c|c|c|c|c|c|}
\hline & & \multicolumn{6}{|c|}{ ANSIEDAD-RASGO } \\
\hline & & \multicolumn{2}{|c|}{ LEVE } & \multicolumn{2}{|c|}{ MODERADO } & \multicolumn{2}{|c|}{ SEVERO } \\
\hline & & Recuen. & $\%$ & Recuen. & $\%$ & Recuen. & $\%$ \\
\hline \multirow{5}{*}{$\begin{array}{c}\text { GRADO } \\
\text { DE } \\
\text { TTM }\end{array}$} & Función normal & 3 & $8,6 \%$ & 1 & $5,0 \%$ & 0 & $0 \%$ \\
\hline & Disfunción leve & 15 & $42,9 \%$ & 14 & $70,0 \%$ & 18 & $72,0 \%$ \\
\hline & $\begin{array}{l}\text { Disfunción } \\
\text { moderada }\end{array}$ & 17 & $48,6 \%$ & 4 & $20,0 \%$ & 4 & $16,0 \%$ \\
\hline & $\begin{array}{l}\text { Disfunción } \\
\text { severa }\end{array}$ & 0 & $0 \%$ & 1 & $5,0 \%$ & 3 & $12,0 \%$ \\
\hline & Total & 35 & $100,0 \%$ & 20 & $100,0 \%$ & 25 & $100,0 \%$ \\
\hline
\end{tabular}

Pruebas de chi-cuadrado de Pearson 0,020 p <0,05 (SIGNIFICATIVO) 


\section{Tabla 11. Niveles de ansiedad rasgo-estado según el grado de TTM}

\begin{tabular}{|c|c|c|c|c|c|c|c|}
\hline & & \multicolumn{6}{|c|}{ ANSIEDAD RASGO-ESTADO } \\
\hline & & \multicolumn{2}{|c|}{ LEVE } & \multicolumn{2}{|c|}{ MODERADO } & \multicolumn{2}{|c|}{ SEVERO } \\
\hline & & Recuen. & $\%$ & Recuen. & $\%$ & Recuen. & $\%$ \\
\hline \multirow{5}{*}{ Grado de TTM } & Función normal & 3 & $8,3 \%$ & 1 & $5,0 \%$ & 0 & $0 \%$ \\
\hline & Disfunción leve & 14 & $38,9 \%$ & 14 & $70,0 \%$ & 19 & $79,2 \%$ \\
\hline & $\begin{array}{l}\text { Disfunción } \\
\text { moderada }\end{array}$ & 18 & $50,0 \%$ & 4 & $20,0 \%$ & 3 & $12,5 \%$ \\
\hline & Disfunción severa & 1 & $2,8 \%$ & 1 & $5,0 \%$ & 2 & $8,3 \%$ \\
\hline & Total & 36 & $100,0 \%$ & 20 & $100,0 \%$ & 24 & $100,0 \%$ \\
\hline
\end{tabular}

Pruebas de chi-cuadrado de Pearson 0,020 p<0,05 (SIGNIFICATIVO)

(moderado y severo), 43 presentaron un grado de TTM. (Ver Tabla No 11)

\section{CONCLUSIONES}

1. La prevalencia de ansiedad en la muestra estudiada fue más predominante en el nivel leve, seguido del nivel severo y moderado.

2. Los niveles de ansiedad en relación al género y la edad no fueron significativos; la tendencia del sexo femenino fue a una ansiedad severa, y la del masculino, a una ansiedad leve. En relación con la edad, todos los individuos tuvieron tendencia a la ansiedad leve, y el grupo de 23 a 26 años, a ansiedad severa.

3. La prevalencia de los TTM fue del orden del $95 \%$. En lo referente a la severidad, el grado leve $(58,8 \%)$ fue el que más se presentó, seguido del moderado $(31,3 \%)$ y el severo (5\%).

4. En relación con el grado de TTM y el género, las diferencias no son significativas, pero se observa cierta tendencia del sexo femenino, a presentar TTM leve y severo en mayor porcentaje que el masculino, en el que prevalece el grado moderado.

5. No se observan diferencias significativas en el grado de TTM y la edad, pero todos los grupos etáreos tienen tendencia a los TTM leve y moderado.

6. Los signos y síntomas de TTM que más se encontraron fueron: movilidad mandibular 75 (93,75\%), alteraciones en la función de la ATM 51 (63,75\%) y dolor en los músculos masticatorios 26 (32,5\%).

7. La presencia de signos y síntomas, según el Índice de Helkimo en relación al género, no es significativa, pero se observa una tendencia de mayor frecuencia en el sexo femenino.

8. Los diferentes niveles de ansiedad tienen una influencia significativa sobre los diversos grados de trastornos temporomandibulares.

\section{DISCUSIÓN DE RESULTADOS}

Los resultados encontrados apoyan el hecho de que los estados de ansiedad tienen una influencia significativa en los individuos para la exacerbación de los TTM. Lo realizado en nuestro trabajo concuerda con Steenks, M.H. en el sentido de que el manejo clínico de los TTM y del dolor orofacial en los casos simples excede en número a los casos más complejos. Por lo tanto, para el odontólogo de prácticageneral, la solución de los casos, en la mayoría reversibles, se apoya preferentemente en el tratamiento rehabilitador oclusal. La fisioterapia puede ser muy acertada en las manos del odontólogo general, es decir, puede 
manejar y dar solución a casos de disfunción leve, que en nuestro estudio llegó a un 58,8\% de la muestra. ${ }^{21}$ Respecto de la prevalencia de ansiedad, se encontró que los niveles de ansiedad leve, moderado y severo tienen cifras muy parecidas tanto para la ansiedad-estado como para la ansiedad-rasgo: (ansiedad-estado leve: 37,5\%, moderado: $26,25 \%$; ansiedad-rasgo leve: 43,75\%, moderado: 25\%, y severo: $31,25 \%$ ). Estas cantidades difieren con las encontradas por Ortiz, F., que halló que la prevalencia de ansiedad-rasgo y estado se concentró en el nivel leve. ${ }^{4}$ Las variables estudiadas de género y grupo etáreo no nos reportaron significancia estadística tanto en su relación con los niveles de ansiedad como con los TTM. En cuanto a los niveles de ansiedad-estado y ansiedad-rasgo, se encontró una tendencia de mayor predisposición en el sexo femenino (ansiedad-estado moderado: 25\%, severo: $39,6 \%$; ansiedad-rasgo moderado: 31,3\%, severo: $33,3 \%)$. Estos resultados son similares a los encontrados por Kleinknecht R. A. et al. (34,8\% de ansiedad moderada y severa) y a los de Ortiz, que encontró un nivel severo de ansiedad correspondiente al 36,8\%, ambos resultados en el sexo femenino. ${ }^{23,4}$ Los resultados de niveles de ansiedad-estado y rasgo según la edad fueron: ansiedad-estado, una leve tendencia hacia un nivel severo en los grupos etáreos de 18 a 22 años $(37,5 \%)$ y de 23 a 26 años (28\%). Estos datos difieren, en relación con el grupo etáreo, de los encontrados por Kleinknecht R. A. et al, que observó el mayor nivel de ansiedad en individuos de 30 a 49 años. ${ }^{22}$ La prevalencia de los TTM encontrada en nuestro estudio fue bastante marcada; de un total de 80 individuos, 76 (95\%) presentaron algún grado de TTM, siendo significativamente mayor que los encontrados por Pedroni, C.R. et al. $(68 \%)$ y Paredes, G. $(85,09) \%$. Respecto de la severidad de los TTM, esta se concentró en el grado leve $(58,8 \%)$, sumando el moderado y severo $36,3 \%$. En lo concerniente al grado de TTM en relación con el género, la disfunción leve y severa predominó en el sexo femenino con $62,5 \%$ y $6,3 \%$ respectivamente, en comparación con el masculino, grupo en el que fue mayor la disfunción moderada $(34,4 \%)$; el sexo femenino presentó grados de TTM en un 97,9\%, y el masculino, en $90,6 \%$. Se puede colegir que estos datos concuerdan con los de Irving, J., Agerberg y Carlson y Agerbergy Osterberg, que encontraron una proporción de 2 a 1 entre el sexo femenino y el masculino. En el grado de TTM según el grupo etáreo, no se encontraron mayores diferencias en los diferentes grupos de edades. Los signos y síntomas asociados a los TTM que en mayor porcentaje se encontraron fueron: movilidad mandibular $(93,75 \%)$, alteraciones de la función de la $\operatorname{ATM}(63,75 \%)$ y dolor muscular $(32,5 \%)$, Los resultados en cuanto a movilidad mandibular y dolor muscular son similares a los reportados por Irving, J. (80\% y 33\% respectivamente). Del mismo modo, nuestros datos de dolor muscular tienen similitud con los de Agerberg y Carlson, Agerberg y Osterberg, que reportaron un porcentaje que va del 13 al 74\%. En relación con los niveles de ansiedad según el grado de TTM, los individuos con niveles de ansiedad-estado, ansiedad-rasgo y ansiedad rasgo-estado severo se presentaron en porcentajes mayores que los de leve y moderado, mostrando, en consecuencia, algún grado de TTM. Estos datos concuerdan con los encontrados por Paredes, G., que reporta diferencias significativas entre la ansiedad y los TTM. 22, 23, 15, 16, 17, 24

\section{REFERENCIAS BIBLIOGRÁFICAS}

1. Castillo Hernández, R.; Reyes Cepeda, A.; Gonzales Hernández, M.; Machado Martínez, M. "Hábitos parafuncionales y ansiedad versus disfunción temporomandibular". Revista cubana de ortodoncia 2001; 16 (1): 14-23

2. Bomjardim, L.R.; Gaviao, M.B; Pererira, L.J.; Castelo, P.M. Anxiety and depresion in adolescents and their relatioship with signs 
and symptoms of témporomandibular disorders. Int J Prosthodont 2005; 18 (4): 347 352

3. John, Mike T.; Reissmann, Daniel R.; Schierz, Oliver; Wassell, Robert W. Oral HealthRelated Quality of Life in Patients With Temporomandibular Disorders. Jornal of Orofacial Pain 2007; 21 (1): 46-54

4. Ortiz Culca, Fernando. Patrón de contacto oclusal en posiciones intermedias y su relación con los trastornos témporomandibulares en individuos jóvenes con dentición natural completa [Tesis para optar el grado académico de magíster en estomatología] Lima, UNMSM; 2003, pág. 92,93

5. Geososalud.com [Revista virtual] 2007 [Fecha de acceso 24 de abril de 2007] En: http://geosalud.com/Salud\%20Ocupacional/estres laboral.htm

6. Molina, F.M. Prevalencia de disfunción cráneomandibular según el indice Helkimo, en la Escuela de Oficiales de la PNP en el periodo octubre 93 - enero 94 [Tesis para optar el título de cirujano dentista] Lima, UNMSM; 1994

7. Schulte, J.K.; Anderson, G.C.; Hathaway, K.M.; Hill, T.E.Psychometric profiles and related pain characteristics of temporomandibular disorder patients. J Orofacial Pain 1993; 7: 247-253

8. González de River, J.L.; De la Hoz, J.L.; Rodríguez-Abaín, M.J.; Monterrey, A.L. Perfil psicopatológico en pacientes con disfunción temporomandibular. An Psiquiatr 1999; 3: 91-95

9. Greene, C.S,; Marbach, J.J. Epidemiologic studies of mandibular dysfunction. A critical review. J Prosthet Dent 1982; 48 (2): 184190

10. Mc Neill, C. History and evolution of TMD concepts. Oral Surgery, Oral Medecine, Oral Pathology, Oral Radiology and Endodontics 1997; 83; 51-60

11. Pullinger, A.G,; Seligman, D.A. Quantifica- tion and validation of predictive values of oclusal variables in temporomandibular disorders using a multifactorial analysis. Journal of Prosthetic Dentistry 2000; 83 (1): 66-75

12. Laskin, D.M. Diagnosis and etiology of miofacial pain and dysfunction. Medical Management of Temporomandibular Disorders 1995; 7 (1): 73-78

13. Moulin, C. From bite to mind: A personal and literature review. The International Journal of Prosthodontics 1999; 12 (3): 279-288

14. National Institutes of Health Tecnology Assesment Conference Statement, april 29-may 1, 1996 (1997). Management of temporomandibular disorders. Oral Surgery, Oral Medecine, Oral Pathology, Oral Radiology and Endodontics; 83: 177-183

15. Irving, J.; Word, G.D.; Hackett, A.F. Does temporomandibular disorder pain dysfunction síndrome affect dietary intake? Dent Update 1999; 26 (9): 405-407

16. Agerberg, G., Carlson, G. Functional disorders of the masticatory system: Idistribution of symptoms by questionnaire. Act Odont Scand 1972; 30 (6): 597-613

17. Agerberg, G.; Osterberg, T. Maximal mandibular movements and symptoms of mandibular dysfunction in 70 years old man and woman. Swed Dent J 1974; 76 (5): 147-163

18. Díaz Fernández, J.M.; Velásquez Bleg, R.; Alfonso Reyes, H. Efecto del tratamiento quirúrgico de los terceros molares inferiores sobre el síndrome de disfunción temporomandibular. Rev Cubana Estomatol 1996; $32(2): 76$

19. Estrella, G. Detección precoz de los desórdenes temporomandibulares. Bogotá, Panamericana Formas e impresos S.A.; 2006 Pag. 107, 108,95

20. Maglione, Horacio O.; Laraudo, Jorge; de Zavala, Luis. Disfunción craneomandibular. Afecciones de los músculos masticatorios y de la articulación temporomandibular. Dolor orofacial. Diagnóstico y terapéutica. Bo- 
gotá, Actualidades Médico-Odontológicas Latinoamérica; 2008, pág. 122, 144, 95, 96

21. Steenks. M.H. The gap batwen education and clinical treatmente in témporomandibular disorders and orofacial pain. Journal of Oral Rehabilitation 2007; (7): 475-477

22. Kleinknecht R.A. Mahoney E.R.; Alexander I.D.. Psychosocial and demographic correlates of temporomandibular disorders and related symptoms: An assessment of community and clinical finding. Pain 1987: 29 (3) 313-24
23. Pedroni, C.R; De Oliveira, A.S; Guarantini, M.I. Prevalence study of sings symptoms of témporomandibular disorders in university students. Journal of Oral Rehabilitation 2003; 30 (3): 283-389

24. Paredes Coz, Gerson. Disfunción craneomandibular y ansiedad en las áreas de influencia de la Facultad de Odontología de UNMSM [Tesis para optar el grado académico de magíster en estomatología] Lima: Universidad Nacional Mayor de San Marcos; 1998, pág. 39, 40 